\title{
Analysis of Magnetic Field and Geometry Effects for the Design of HTS Devices for AC Power Applications
}

\author{
Francesco Grilli, Luciano Martini, Svetlomir Stavrev, Bertrand Dutoit, and Roberto Brambilla
}

\begin{abstract}
The performance of HTS devices is strongly influenced by local values of the current and field distributions. In this paper, we investigate the influence of the magnetic field and the geometrical configuration on the loss behaviour of a $200 \mathrm{kVA}$ FCL prototype, composed by Bi-2223/Ag tapes wound around a cylindrical support. The investigation is performed by means of finite element computations, with the use of an axisymmetric 2D A-V formulation for taking into account the cylindrical geometry. The electrical behaviour of the superconductor is described by means of a B-dependent E-J power-law relation, derived from experimental measurements with a field of different orientation. Several geometrical configurations are analyzed and compared, in order to find the ones with the lowest $\mathrm{AC}$ loss.
\end{abstract}

Index Terms - AC losses, finite element method, high temperature superconductors, magnetic field distribution

\section{INTRODUCTION}

$\mathbf{F}$ AULT current-limiters (FCL) are one of the most promising applications for exploiting the properties of high$T_{c}$ superconductors (HTS). Since they operate at subcritical current for most of the time, it is very important that they have a reduced AC power dissipation in such nominal conditions, in addition to a very good performance in the limiting phase [1]. The current limitation of a resistive FCL is based on the quench of the HTS when the current exceeds its critical current. This results in an abrupt increase of its electrical resistance and thereby automatically limiting peak short-circuit currents. There are many locations in transmission and distribution grids where FCL can offer technical and economical benefits [2]. Eventually, depending on the specific application, different designs and HTS conductors can be considered [3].

In the framework of an Italian R\&D project, we are developing a $200 \mathrm{kVA} 3$-phase FCL prototype, by using commercially available HTS tapes. In principle, a single-phase FCL consists of a cylindrical insulated support, around which one or more layers of superconducting tape are wound, in a manner to have a large surface in contact with the liquid cryogen bath. It is necessary to dissipate the large amount of power during the

Manuscript received October 3, 2004. Corresponding author's e-mail: fgrilli@lanl.gov.

F. Grilli, S. Stavrev and B. Dutoit are with the Swiss Federal Institute of Technology - Lausanne, 1015 Lausanne, Switzerland. F. Grilli is now with the Los Alamos National Laboratory, MST-STC, Los Alamos, New Mexico 87545, United States. L. Martini and R. Brambilla are with CESI SpA, Via Rubattino 54, 20134 Milano, Italy.

This work is supported partially by the Swiss National Science Foundation through the National Center of Competence in Research "Materials with Novel Electronic Properties - MaNEP" and partially by Research Fund for Italian Electrical System established with Ministry of Industry Decree DM 26/1/2000. limiting phase. Tapes produced by American Superconductor Corporation (AMSC) [4], with self-field critical current of $\sim 120 \mathrm{~A}$ at $77 \mathrm{~K}$, have been used.

In this paper, we have investigated the influence of the tape arrangement on the $\mathrm{AC}$ losses of the device. In particular, the influence of the winding gap between the tapes and the use of a bifilar winding have been studied. For this aim, we have utilized finite-element method (FEM) simulations, which allow precise computation of the current and field distribution, including the $\mathrm{AC}$ losses in the superconductor, and taking into account the local effects of the magnetic self-field [5], [6].

It should be noted that the developed method can be applied for investigating devices other than FCL, such as superconducting transformers and magnets, where the local distribution of the magnetic field and the arrangement of the conductors play an important role.

\section{Finite Element Model fOR HTS CONDUCTORS}

In order to calculate the current and field distribution inside superconductors, we have used the FEM software package FLUX [7]. Due to the intended cylindrical geometry, a 2D axisymmetric model was used. This means that only a crosssection of the geometry in the longitudinal direction was considered. The 2D FEM model of HTS used the magnetic vector potential $\mathbf{A}$ and the electric potential $V$ as state variables. Details can be found in [6]. The HTS is described by means of a non-linear power-law relation $E=E_{c}\left(J / J_{c}(B)\right)^{n(B)}$. The critical electric field $E_{c}$ was $10^{-4} \mathrm{~V} / \mathrm{m}$, where the parameters $J_{c}$ and $n$ depend on the local magnetic field experienced by the HTS material. The metallic sheath is described by a constant conductivity of $5 \cdot 10^{8} \mathrm{~S} / \mathrm{m}$, a typical value for silver at $77 \mathrm{~K}$.

The magnetic field dependence of $J_{c}$ and $n$ has been measured from current-voltage characteristics at $77 \mathrm{~K}$, with applied magnetic fields up to $0.45 \mathrm{~T}$, having varying orientation with respect to the tape surface. The decrease of the critical current $I_{c}$ as a function of the field orientation (parallel and perpendicular to the tape surface) is shown in Fig. 1. The field dependence of the power index $n$ has a similar shape. This figure shows that for fields lower than $0.1 \mathrm{~T}$ the parallel component does not affect the critical current of the tape. In addition, as confirmed by FEM simulations, in our device the magnetic field inside the superconductor never exceeds $0.1 \mathrm{~T}$. Therefore, instead of fitting the data in the entire range of magnetic field with a quite complex mathematical expression, we have preferred to use a simple one, valid in the field range 


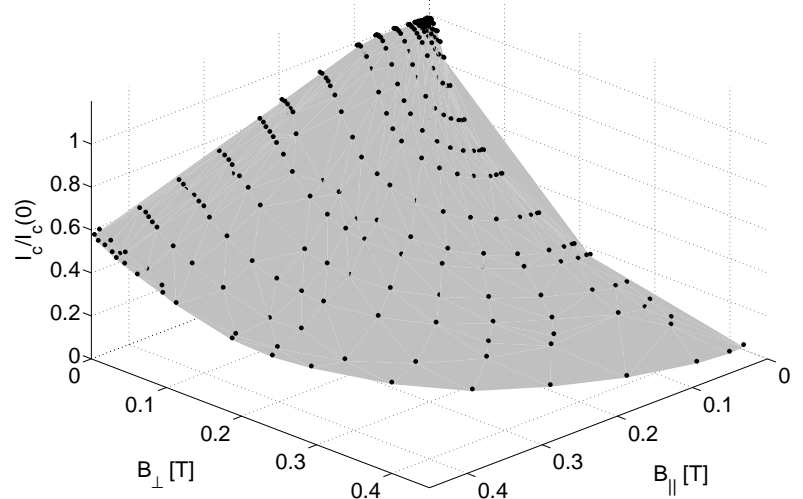

Fig. 1. Measured critical current, normalized by a self field value at $77 \mathrm{~K}$ of $I_{c}(0)=118 \mathrm{~A}$, as a function of the parallel and perpendicular magnetic field components. The grey surface is a guide for the three-dimensional view.

of interest. In this way, $J_{c}(B)$ and $n(B)$ models dependent only on the perpendicular component of the field can be used.

From the fitting of the experimental measurements in the range between zero and $0.1 \mathrm{~T}$, we have derived the following $J_{c}(B)$ and $n(B)$ models [8]:

$$
\begin{aligned}
J_{c}(B) & =\frac{J_{c 0}}{23.7-22.7 e^{-B_{\perp} / B_{0}}} \\
n(B) & =\frac{n_{0}}{20.05-19.05 e^{-B_{\perp} / B_{0}}}
\end{aligned}
$$

where $J_{c 0}$ and $n_{0}$ are HTS material characteristics in the absence of any magnetic field (self or external), $B_{\perp}$ is the absolute value of the perpendicular magnetic field component (in $\mathrm{T}$ ), and $B_{0}$ is a normalizing constant of $1 \mathrm{~T}$, formally needed to obtain dimensionless exponents. The parameter $J_{c 0}$ is higher than the value obtained by dividing the self-field critical current by the HTS cross-section [9].

\section{AC LOSSES OF TAPES: MEASUREMENTS AND SIMULATIONS}

In order to verify the FEM calculations, we compared computed and measured $\mathrm{AC}$ losses on $10 \mathrm{~cm}$ straight samples. The FEM geometry has been drawn by starting from a picture of typical cross-section of BSCCO multifilamentary tapes of this kind. Since the HTS filaments are tightly packed, we simulated a mono-filamentary tape with an elliptical crosssection, maintaining the same aspect ratio of the HTS area and the distance from the edge as in the real tape. Both are important factors that influence the self-field $\mathrm{AC}$ losses in mono-filamentary conductors.

The losses have been measured at $59 \mathrm{~Hz}$ by a standard electrical characterization technique (see for example [10]), both with two voltage taps soldered onto the tape and by means of a contactless rectangular loop positioned beneath the tape. Figure 2 shows the measured losses as a function of the transport current, compared to the results of FEM simulations and to the prediction of Norris's analytical models [11]. The

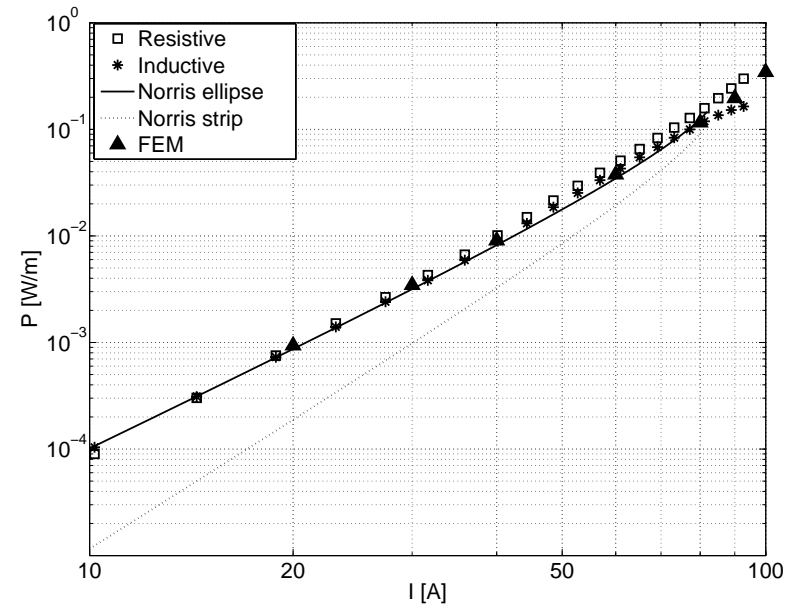

Fig. 2. Measured and computed AC losses at $59 \mathrm{~Hz}$ as a function of transport current (rms values) for a multifilamentary BSCCO tape made by AMSC.

experimental AC losses fall close to the prediction for the ellipse. For FEM simulations, we have used a $J_{c 0}$ value of $1.7 \cdot 10^{8} \mathrm{~A} / \mathrm{m}^{2}$ and a power index $n_{0}$ equal to 19 . This value of $J_{c 0}$ produces an effective critical current $\left(I_{c}\right)$ of $118 \mathrm{~A}$, which can be computed by integrating the local values of $J_{c}(B)$ on the HTS cross-section [9]. The losses in the HTS were computed as follows:

$$
P=\frac{1}{T} \int_{0}^{T} d t \int_{S} \mathbf{J} \cdot \mathbf{E} d S \quad[\mathrm{~W} / \mathrm{m}]
$$

where $T=1 / f$ is the second simulated period (to avoid transient terms), $f$ is the frequency of the transport current $(59 \mathrm{~Hz})$ and $S$ is the HTS cross section. As it can be seen from Fig. 2, the computed losses (full triangles) were close to the measured values.

\section{AC LOSS PREDICTION FOR FCL APPLICATIONS}

\section{A. Single layer configuration}

The FCL will consist of a large number of turns $(\mathrm{N}>100)$ around a cylindrical support. Due to the impossibility of simulating the whole geometry, we first determined how many turns had to be simulated in order to make a fair estimate of the behaviour of the central part of the device. For a working condition, we chose an operating transport current of $80 \mathrm{~A}_{\mathrm{rms}}$ at $59 \mathrm{~Hz}$.

We computed the AC losses for various numbers of turns $(\mathrm{N}), \mathrm{N}=1,3,5, \ldots, 11$. By increasing the number of turns, the central tapes are progressively shielded from the penetration of the perpendicular magnetic flux, and exhibit lower AC losses (Fig. 3a). The decrease of the losses of the central tape as a function of the number of simulated turns is shown in Fig. 3b: the losses of the central tape tend to become stable for a sufficiently large number of simulated turns. As a consequence we have chosen the central tape of the configuration with $\mathrm{N}=7$ as a good approximation for describing the behaviour of the central part of the FCL in the real situation. The behaviour of the turns at the ends of the winding cannot be described with 

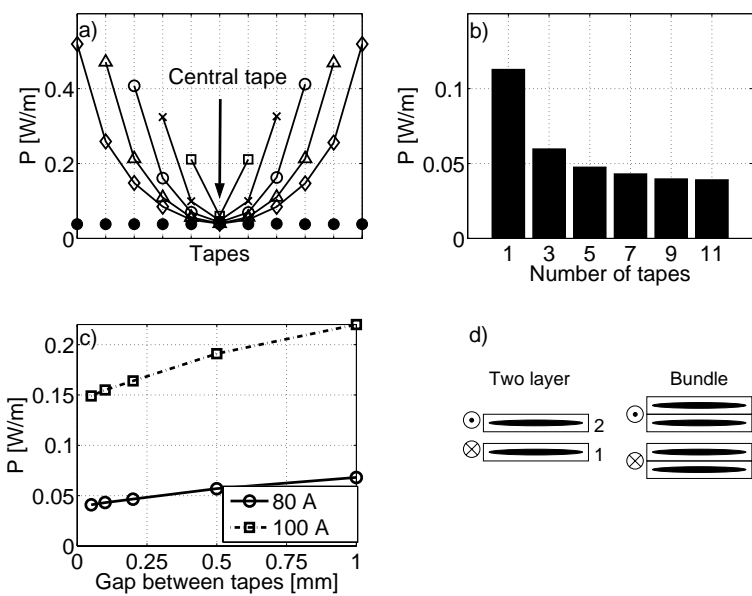

d)

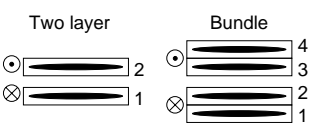

Fig. 3. a) Calculated AC losses in HTS tapes for different numbers of turns. The full symbols correspond to the bifilar configuration (subsection IV-B). b) $\mathrm{AC}$ losses in the central tape as a function of the number of simulated tapes. c) AC losses in the central tape for different values of the gap between the windings. d) Two-layer and bundle configurations analyzed in subsections IV-B and IV-C.

such an approximation, because it strongly depends on the number of turns; on the other hand, the difference between the central part and the ends disappears for bifilar configurations with current flowing in opposite directions, see subsection IVB.

We also studied AC loss dependence on the gap between the tapes at two current rates: $80 \mathrm{~A}_{\mathrm{rms}}$ and $100 \mathrm{~A}_{\mathrm{rms}}$. The results are displayed in Fig. $3 \mathrm{c}$ for different values of the gap. Increasing the gap value from $0.05 \mathrm{~mm}$ to $1 \mathrm{~mm}$ resulted in a significant increase of the losses, about 50\%. This is due to the penetration of the magnetic flux between the tapes, which results in a larger perpendicular component of the magnetic field acting on the superconductor, especially at the tape edges, where most of the current flows.

\section{B. Two-layer configuration}

The single-layer configuration considered so far has two main disadvantages: firstly, it makes the winding quite long (L>1 m per phase); secondly, it has large losses at the ends (see Fig. 3a) due to the large perpendicular field component there. Therefore, it is interesting to investigate the possibility of eliminating this effect with a bifilar winding (see Fig. 3d). This configuration reduces the perpendicular component of the field and allows a more compact device for the same quantity of HTS tape. These effects have been experimentally studied in [12]. Here, we evaluate quantitatively the reduction of the AC losses by means of FEM simulations.

The presence of the second layer leads to uniform losses in the different windings, avoiding the large increase of the losses near the two ends of the device (see the full circles in Fig. 3a). Furthermore, the losses in the central tape were slightly smaller. At $80 \mathrm{~A}_{\mathrm{rms}}$ the losses in the central part of the winding were reduced from $4.31 \cdot 10^{-2} \mathrm{~W} / \mathrm{m}$ to $3.83 \cdot 10^{-2}$ $\mathrm{W} / \mathrm{m}$, at $100 \mathrm{~A}_{\mathrm{rms}}$ from $15.5 \cdot 10^{-2} \mathrm{~W} / \mathrm{m}$ to $9.98 \cdot 10^{-2} \mathrm{~W} / \mathrm{m}$,

\section{a) Single layer}

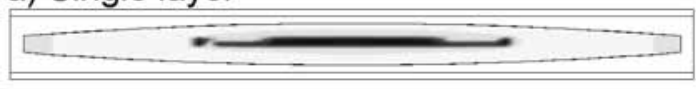

b) Two layer

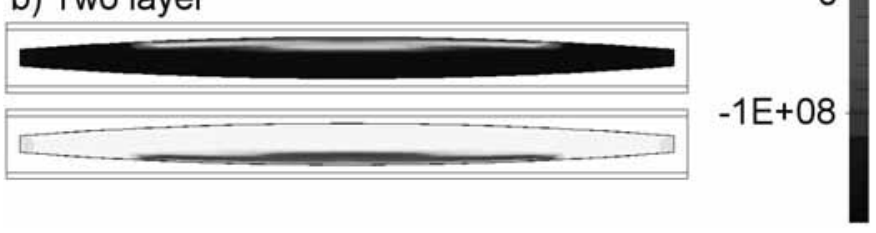

Fig. 4. Current density distribution at peak current of $80 \mathrm{~A}_{\mathrm{rms}}$ : a) singlelayer and b) two-layer configuration.

corresponding to $11 \%$ and $36 \%$ reductions. The second layer also caused deep changes in the shape of the internal current density distribution. Due to the magnetic coupling between the two layers, the current distribution in each tape is no longer fully symmetric, and most of the current tends to flow in the part of the tape closest to the adjacent tape (Fig. 4).

\section{Bundle configuration}

In order to attain a HTS device with higher current rates, one can use two opposite layers, but each of them composed by two (or more) HTS tapes in parallel (bundle), as indicated in Fig. 3d. The current evolution and the AC losses were studied for two different cases: 1) letting the total current free to distribute across the two tapes, 2) forcing the currents to be equal. With the FLUX software, the two cases are obtained by connecting the tapes of the bundle in parallel or in series in the electric circuit, respectively. As shown in Fig. 5a, in the parallel case, the current tends to flow as long as possible in the central tapes of the two bundles (tapes 2 and 3 in Fig. 3d). This rapidly saturated them, leaving the other two tapes ( 1 and
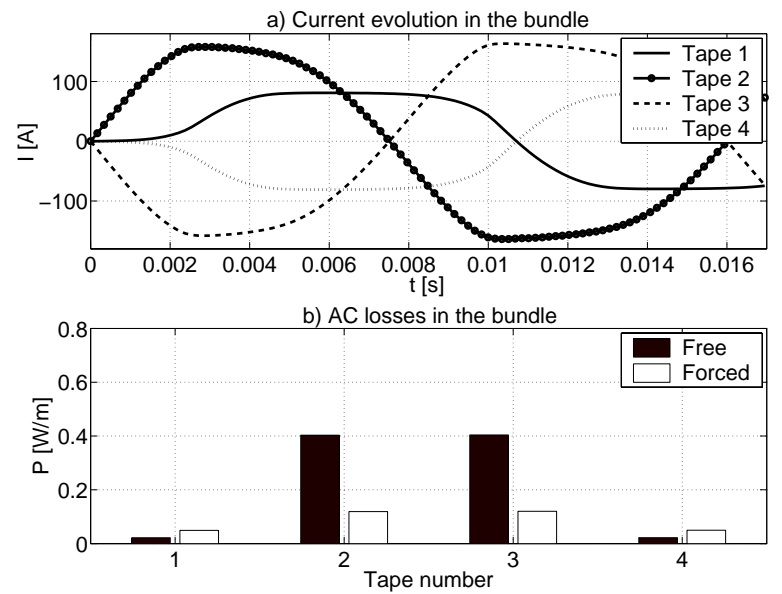

Fig. 5. a) Current evolution in the four tapes composing the two bundles, when a current of $160 \mathrm{~A}_{\mathrm{rms}}$ is left free to distribute across the two tapes of each bundle. b) AC losses comparison: current left free to distribute between the two tapes of the bundle or forced to be equal in each tape. 


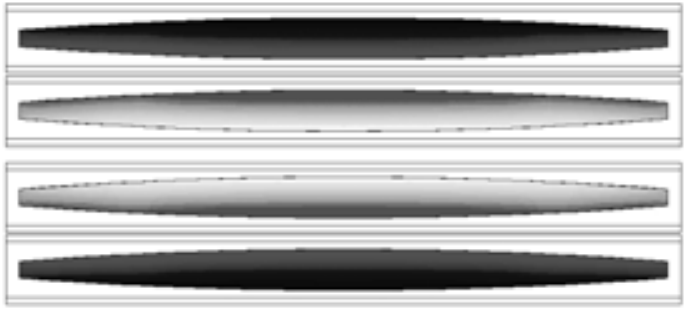

Fig. 6. Distribution of the parallel component of the magnetic field in the bundle configuration at the peak value of a transport current of $160 \mathrm{~A}_{\mathrm{rms}}$ equally shared between the two tapes of each bundle. The internal tapes are subjected to a magnetic field of about $60 \mathrm{mT}$.

4) scarcely utilized. This current distribution leads to nonsinusoidal current waveforms and to large AC losses in the saturated internal tapes as can be seen in Fig. 5b (black bars).

When the current is equal in each tape, saturation is avoided, the current is sinusoidal, and the losses are consequently much smaller (Fig. 5b, white bars). Nevertheless, also in this case the losses of tapes 2 and 3 are higher than the ones of tapes 1 and 4 , due to the fact that the inner layers experience a higher $d B / d t$, which causes larger ohmic dissipation and hysteresis losses in the superconductor. The distribution of the magnetic field is shown in Fig. 6.

\section{Summary AND CONCLUSIONS}

In this work, finite element simulations have been used for computing the current and field distributions, and evaluating the AC losses in a superconducting fault-current-limiter during nominal operating conditions. The $J_{c}(B)$ relation used in the simulations was derived from experimental measurements of the current-voltage characteristics in applied magnetic field with varying orientation. The finite-element model was tested by comparing the measured and computed self-field AC losses of short-tape samples. Finally, a cylindrical HTS winding of a FCL was modelled. The influence of different geometrical parameters and configurations on the total AC loss behaviour was investigated.

We found that the distance between the HTS turns plays an important role, and the gap between the tapes must be reduced to at least $0.2 \mathrm{~mm}$ in order to minimize the magnetic flux penetration in between.

Bifilar windings were very effective for reducing the losses at the ends of the device and to make them uniform along the turns. The bifilar winding allows a more compact geometry, with less losses in the central part of the device.

Tape bundles in parallel connection do not distribute current evenly. Despite the large available superconducting section, the current is not equally shared between the tapes but, due to magnetic coupling, is forced to flow in the central tapes easily causing saturation phenomena with consequent increase of the AC losses. Practical solutions for avoiding such saturation phenomena by means of a more uniform current repartition between the tapes of the bundle have to be searched within manufacturing limits.

The FEM analysis is an effective tool to analyze the electromagnetic behaviour of complex devices involving superconductors. Coupling the electromagnetic and thermal models is needed to predict overcritical current response. The choice of the mathematical formulation, the mesh grid, and the order and type of shape functions underlying the finite elements modelling are the key issues and the challenge for obtaining a reliable model.

\section{REFERENCES}

[1] R.F. Giese, "Fault Current limiters", Handbook of superconducting materials", Institue of Physics Publishing, pp. 1625-1632, 2003.

[2] W. Paul et al., "Fault current limiter based on high temperature superconductor - different concepts, test results, applications, simulations", Physica C, vol. 354, pp- 27-33, 2001.

[3] M. Noe and B.R. Oswald, "Technical and economical benefits of superconducting fault current limiters in power systems", IEEE Transactions on Applied Superconductivity, vol. 9, no. 2, pp. 1347-1350, 1999.

[4] American Superconductor Corporation, http://www.amsuper.com

[5] N. Amemiya et al., "Numerical analysis of AC losses in high Tc superconductors based on E-j characteristics represented with n-value Applied Superconductivity," IEEE Transactions on Applied Superconductivity, vol. 7, no. 2, pp.2110-2113, June 1997.

[6] S. Stavrev et al., "Comparison of numerical methods for modeling of superconductors", IEEE Transactions on Magnetics, vol. 38, no. 2, pp. 849-852, Mar. 2002.

[7] Cedrat SA, Meylan, France, http://www.cedrat.com

[8] S. Stavrev, B. Dutoit, and N. Nibbio, "Geometry considerations for use of Bi-2223/Ag tapes and wires with different models of $\mathrm{J}_{\mathrm{C}}(\mathrm{B})$ ", IEEE Transactions on Applied Superconductivity, vol. 12, no. 3, pp. 18571865, Sept. 2002.

[9] S. Stavrev, B. Dutoit, and F. Grilli, "Self-field and geometry effects in transport current applications of multifilamentary Bi-2223/Ag conductors", IEEE Transactions on Applied Superconductivity, vol. 13, no. 3, pp. 3807-3813, Sept. 2003.

[10] F. Gömöry et al., "Contactless measurement of hysteretic transport AC losses in multifilamentary BiSrCaCuO-2223/Ag tapes", Physica C, vol. 308, pp. 203-214, 1998.

[11] W.T. Norris, "Calculation of hysteresis losses in hard superconductors carrying ac: isolated conductors and edges of thin sheets," Journal of Physics D: Applied Physics, vol. 3, pp. 489-507, 1970.

[12] S. Spreafico et al., "The effect of self-field on current capacity in Bi-2223 composite strands", IEEE Transactions on Applied Superconductivity, vol. 9, no. 2, pp. 2159-2162, June 1999. 